\title{
The symmetry problem: current theories and prospects
}

\author{
Richard Breheny $^{1}$ - Nathan Klinedinst ${ }^{1}$. \\ Jacopo Romoli ${ }^{2}$ (I) Yasutada Sudo ${ }^{1}$
}

\begin{abstract}
The structural approach to alternatives (Katzir in Linguist Philos 30(6):669_ 690, 2007; Fox and Katzir in Nat Lang Semant 19(1):87-107, 2011; Katzir in Semantics, pragmatics and the case of scalar implicatures, Palgrave Macmillan, London, pp 40-71, 2014) is the most developed attempt in the literature at solving the symmetry problem of scalar implicatures. Problematic data with indirect and particularised scalar implicatures have however been raised (Romoli in Snippets 27:14-15, 2013; Trinh and Haida in Nat Lang Semant 25(4):249-270, 2015). To address these problems, Trinh and Haida (2015) proposed to augment the theory with the Atomicity Constraint. Here we show that this constraint falls short of explaining minimal variants of the original problems, and moreover that it runs into trouble with the inferences of sentences involving gradable adjectives like full and empty. We furthermore discuss how the structural approach suffers at times from the problem of 'too many lexical alternatives' pointed out by Swanson (Linguist Philos 33(1):31-36, 2010), and at other times from the opposite problem of 'too few lexical alternatives'. These three problems epitomise the challenge of constructing just enough alternatives under the structural approach to solve the symmetry problem in full generality. Finally, we also sketch another recent attempt at solving the symmetry problem, Bergen et al. (Semant Pragmat 9(20), 2016), which is based on relative informativity and complexity. We argue that Bergen et al. do not provide a general solution to the symmetry problem either, by pointing to some of the open problematic cases that remain for this approach
\end{abstract}

\footnotetext{
$凶$ Jacopo Romoli jacopo.romoli@gmail.com

Richard Breheny r.breheny@ucl.ac.uk

1 University College London, Chandler House, 2 Wakefield Street, London WC1N1PF, UK

2 Ulster University, Shore Road, Newtownabbey, Belfast BT370QB, UK
} 
as well. We conclude that while important progress has been made in the theory of alternatives for scalar implicatures in the last few years, a full solution to the symmetry problem has not yet been attained.

Keywords Alternatives $\cdot$ Exhaustification $\cdot$ Scalar implicature $\cdot$ Symmetry problem

\section{Introduction}

\subsection{The symmetry problem}

Theories of scalar implicatures, while quite diverse, tend to have the following shape: the scalar implicatures of sentence $S$ are the negations of alternative sentences (or simply alternatives) of $S .{ }^{1}$ In particular, as a first approximation, the scalar implicatures of $S$ are the negation of all alternatives of $S$ that are relevant and more informative than $S .^{2}$ For instance, where (1a) has the scalar implicature in (1b), this is derived in reference to the alternative John did all of the homework.

a. John did some of the homework.

b. $\rightsquigarrow$ John didn't do all of the homework.

A theory of alternatives that explains how alternatives are determined for a given sentence is therefore a crucial part of a theory of scalar implicatures.

An important problem for a theory of alternatives is the so-called symmetry problem, which has to do with the question of how to restrict alternatives (Kroch 1972; Fox 2007; Katzir 2007; Fox and Katzir 2011, among others). In order to see the problem, let us consider a concrete example. For (1a) above, for example, we have to make sure that (2) must not be an alternative.

John did some but not all of the homework.

The reason is that if (2) were an alternative, it would generate its negation as a scalar implicature, namely, that John did either none or all of the homework. Since the literal meaning of (1a) says that John did some of the homework, it would, then, follow that John did all of the homework. However, this scalar implicature would contradict

\footnotetext{
1 There are approaches to scalar implicatures that do not make use of alternative sentences, e.g. van Rooij and Schulz $(2004,2006)$ and Fine (2017). They have, however, equivalent formulations that do (see Spector 2016 for discussion). Our focus in this paper are the approaches explicitly based on alternatives, especially the structural approach to alternatives. We leave for future research investigating how many of the problems discussed here extend to the approaches not explicitly based on alternatives.

2 More precisely, in the neo-Gricean tradition, scalar implicatures are derived in two stages. A primary inference is that the speaker is not certain about any more informative, relevant alternatives. This 'uncertainty' implicature about the speaker is made on the grounds that they will say the most informative relevant thing they believe to be true. A secondary assumption of opinonatedness yields the stronger implicature that the speaker thinks the alternative is not true (see Soames 1982; Horn 1972, 1989; Gazdar 1979; Sauerland 2004, among others). Alternatively, if the speaker is not assumed to be opinionated, an inference that the speaker is ignorant about stronger alternatives is derived. Other approaches to scalar implicatures (Chierchia et al. 2012; Fox 2007, among others) derive the strong scalar implicature directly via a grammatical mechanism that is independent of the mechanisms of ignorance implicature.
} 
the scalar implicature (1b). Thus, in order to explain why (1a) has (1b) as a scalar implicature, it needs to be explained why (2) does not enter the computation of scalar implicatures.

More generally, $S$ with a scalar implicature that $\neg A$ for some alternative $A$ should not have a sentence that means $S \wedge \neg A$ as an alternative. This is because if $S \wedge \neg A$ were an alternative, it would yield a scalar implicature that $\neg(S \wedge \neg A)$, which would contradict what $S$ says and the scalar implicature $\neg A$. We follow the literature and call alternatives like $A$ and $S \wedge \neg A$ symmetric alternatives. ${ }^{3}$

The symmetry problem is, therefore, the problem of how to make sure that $A$ is an alternative to $S$ but not its symmetric alternative $S \wedge \neg A$. It should also be noticed that the fact that (1a) never implicates that John did all the homework implies that (2) cannot be an alternative to the exclusion of John did all of the homework. The theory of alternatives needs to explain this as well.

\subsection{Non-weaker alternatives}

The symmetry problem suggests that the set of alternatives should be somehow restricted. To make the problem more complicated, there are reasons to believe that the set should be able to contain alternatives that are logically independent of the assertion, in addition to alternatives stronger than it. That is, scalar implicatures would arise as the negations of all relevant alternatives that are not entailed by the assertion (i.e. all alternatives that are stronger than the assertion and those that are logically independent from it).

One argument comes from the observation that a non-monotonic operator $O$ embedding a scalar term like some, $O$ (some), can implicate the negation of $O$ (all) (cf. Chemla and Spector 2011; Spector 2007). Importantly, $O$ (all) is a logically independent alternative to $O$ (some).

To see this concretely, consider (3a), involving the non-monotonic operator every letter but $A$. The relevant logically independent alternative is (3b).

a. Every letter but A is connected to some of its circles.

b. Every letter but A is connected to all of its circles.

$\overline{3}$ The definition of symmetric alternatives from Fox and Katzir (2011) is as follows:

(i) Let $S, S_{1}, S_{2}$ be sentences such that $S_{1}$ and $S_{2}$ are alternatives to $S$. We say that $S_{1}$ and $S_{2}$ are symmetric alternatives of $S$ if both of the following are true:

a. $\quad \llbracket S_{1} \rrbracket \cup \llbracket S_{2} \rrbracket=\llbracket S \rrbracket$

b. $\quad \llbracket \mathrm{S}_{1} \rrbracket \cap \llbracket \mathrm{S}_{2} \rrbracket=\emptyset$

Trinh and Haida (2015) modify this version slightly as in (ii) below to extend it to the cases of particularised implicatures that they discuss. Notice that the two definitions are equivalent for a sentence $S$ and alternatives $A$ and $S \wedge \neg A$, if $A$ asymmetrically entails $S$. See also Katzir (2014) for a generalised definition of symmetry.

(ii) a. $\llbracket \mathrm{S}_{1} \rrbracket$ is relevant iff $\llbracket \mathrm{S}_{2} \rrbracket$ is relevant

b. $\llbracket \mathrm{S}_{1} \rrbracket \cap \llbracket \mathrm{S}_{2} \rrbracket=\emptyset$ 

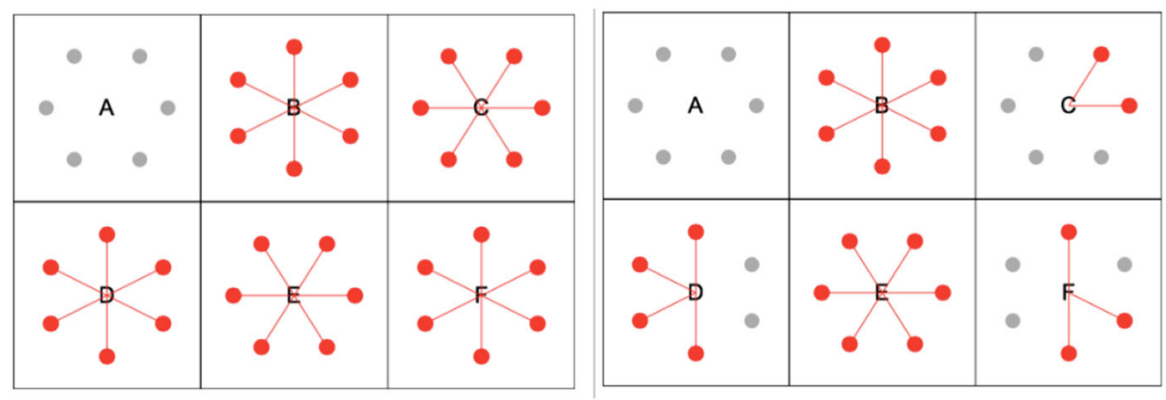

Fig. 1 Situations in which only the literal meaning of (3a) is true (left) and both the literal meaning and the meaning with the inference are true (right)

Assuming that scalar implicatures are cancellable, there are two potential readings for (3a):

(4) a. Reading without the scalar implicature: A is connected with none of its circles; all the other letters are connected with some or all of their circles.

b. Reading with the scalar implicature: A is connected with none of its circles; all the other letters are connected with some of their circles, but not all of these other letters are connected with all of their circles.

These two readings can be distinguished by the connections that the letters other than $A$ have. Concretely, consider the two situations in Fig. 1. The picture on the left only makes (4a) true, while the picture on the right makes both (4a) and (4b) true. The difference between these situations is intuitively clear: (3a) is harder to accept as a description of the picture on the left than of the one on the right. These judgments suggest that the reading with a scalar implicature $(4 \mathrm{~b})$ is available.

Another argument for including logically independent alternatives comes from contextually-determined alternatives. We will discuss such an example below in (11), where John smoked pot counts as an alternative to John ran.

For these reasons, we will assume throughout this paper that the set of alternatives includes not only logically stronger alternatives but also logically independent alternatives. Note that with logically independent alternatives, we will have to deal more often with symmetric alternatives. That is, for a given sentence $S$ with a scalar implicature $\neg A$ based on an alternative $A$, the alternative that means $\neg A$ should not be used to generate a scalar implicature, as it would contradict the observed scalar implicature. For instance, (5) should not be an alternative to (1a).

John did not do all of the homework.

\subsection{Outline}

A well-known method for avoiding the symmetry problem is to assume that alternatives are restricted lexically (Horn 1972, among others). For instance, assuming that all is a lexically specified alternative to some while some but not all is not, we can successfully 
prevent some but not all from entering the computation of scalar implicatures for (1a). This theory, however, does not lead to a deep explanation of why certain items are alternatives and others are not. In the current literature, a more principled explanation has been proposed, namely, the structural approach (Katzir 2007; Fox and Katzir 2011). This paper focuses critically on this approach, its advantages, and remaining open issues. We also discuss briefly an alternative recent approach based on a notion of informativity and cost (Bergen et al. 2016).

The rest of this paper is structured as follows. In Sect. 2, we review how the structural approach to alternatives solves the symmetry problem with examples like (1a), and its problems pointed out by Romoli (2013) and Trinh and Haida (2015). In Sect. 3, we critically discuss the proposal by Trinh and Haida (2015) to augment the structural approach with the Atomicity Constraint, and point out further problems it fails to address. In Sect. 4, we discuss two additional problems for the structural approach - the problem of too many alternatives, and the problem of too few alternatives - which further illustrates the general difficulty of obtaining an adequate set of alternatives under the structural approach. Section 5 is devoted to a brief discussion the abovementioned alternative account of the symmetry problem, the approach based on informativity and cost by Bergen et al. (2016). We argue that this account also does not provide a full solution to the symmetry problem. We conclude the paper in Sect. 6.

\section{The structural approach to alternatives and its problems}

\subsection{The structural approach}

The structural approach to alternatives, advocated by Katzir (2007) and Fox and Katzir (2011), is intended to solve the symmetry problem with a general algorithm for constructing alternatives. The key idea behind it is that alternatives cannot be of greater structural complexity. To illustrate, some and all are legitimate alternatives, as they are of equal structural complexity, while some and some but not all are not, as the latter is more complex.

To be more precise, the set of formal alternatives is defined as in (6).

(6) The set of formal alternatives $F(S)$ of sentence $S$ in context $c$ is the set of sentences derivable by successive replacement of constituents of $S$ with items in the substitution source of $S$ in $c$.

Katzir (2007) defines substitution source as in (7). ${ }^{4}$

(7) An item $\alpha$ is in the substitution source of $S$ in $c$ if

a. $\quad \alpha$ is a constituent that is salient in $c$ (e.g. by virtue of having been mentioned); or

b. $\alpha$ is a subconstituent of $S$; or

c. $\alpha$ is in the lexicon.

\footnotetext{
${ }^{4}$ Fox and Katzir (2011) further employ focus relativity, something we suppress except where relevant; see below.
} 
The substitution source contains items in the lexicon. So, for instance, one could in principle replace, say, a verb with any other verb in the lexicon. As it stands, if all formal alternatives were always employed to derive scalar implicatures, the theory would lead to massive overgeneration, which is clearly undesirable. To address this issue, Fox and Katzir (2011) assume that the context specifies a subset $C$ of $F(S)$, which is meant to represent contextually relevant alternatives. In particular, $C$ is assumed to respect the closure condition in $(8):^{5}$

\section{Closure condition on $C$ :}

a. $C \subseteq F(S)$

b. $S \in C$

c. There is no $S_{0} \in F(S) \backslash C$ s.t. the meaning of $S_{0}$ is in the Boolean closure of $C$.

The first clause requires $C$ to be a subset of the formal alternatives, and the second clause demands what is asserted to be in the set $C$. The third clause, finally, requires $C$ to include all formal alternatives that express Boolean combinations of the meanings of sentences in $C{ }^{6}$

The intuition behind (8) is the following. If it is of interest in the context whether $p$ is true or false, then it is of interest whether $\neg p$ is. Thus, if the former is included, then so should be the latter, unless there is no formal alternative expressing it. Similarly, if it is relevant in the context whether $p$ is true and whether $q$ is true, then it will be relevant whether their conjunction is. Therefore, no formal alternative that belongs to the Boolean closure of $C$ should be left out.

Finally, the scalar implicatures of $S$ are computed by negating the members of $C$ that are not weaker than $S$. More precisely, we will assume the following computation of scalar implicatures (Sauerland 2004; Fox 2007, among others): ${ }^{7}$

(9) Scalar implicatures: The scalar implicatures of $S$ are the negations of all alternatives $A$ of $S$ such that:

a. $A$ is an element of $F(S)$ and $A$ is RELEVANT and NOT WEAKER than $S$, and

b. $\neg A$ does not contradict the negation of any relevant $A^{\prime}$ element of $F(S)$ given $S$.

Now, let us illustrate this theory with (1a). Crucially, the problematic alternative (2) is excluded because it's not in the substitution source: it is not a lexical item, a subconstituent of (1a), and it wasn't mentioned in the context. That is, (2) is not in $F$ (1a)

\footnotetext{
5 Condition (8) is not the only condition on $C$ that has been put forward in the literature. Fox and Katzir (2011) use a notion of exhaustive relevance, Katzir (2014) discusses the option of pruning inferences after exhaustification, and Crnic et al. (2015) propose a different non-weakening constraint. We will stick to (8) because, as far as we can see, the choice here would be inconsequential for our arguments below. We thank Roni Katzir (p.c.) for discussion on this point.

6 The Boolean closure of $C$ is the smallest set $P$ of propositions that contains the propositions expressed by the members of $C$, and whenever $p, q \in P$, we have $\neg p,(p \wedge q) \in P$.

7 (9) is actually a simplified version of what in Fox (2007) is called 'innocent exclusion'. The refined final version used in Fox 2007 requires a second-order quantification over sets of alternatives. We will stick to the simpler (9) as it is enough for our purposes. For discussion see Fox (2007).
} 
(see fn. 8 below about contextual alternatives). On the other hand, some is a lexical item that can be replaced with all and therefore John did all of the homework will be a formal alternative.

These formal alternatives are now restricted to a subset $C$ that obeys (8). For instance, (10) is a permissible set $C$ of relevant alternatives for (1a) and will yield the attested scalar implicature that John did not do all of the homework.

$$
C=\{\text { John did some of the homework, John did all of the homework }\}
$$

Importantly, although the meanings of (2) and (5) are in the Boolean closure of $C, C$ nonetheless obeys (8), since they are not in $F(1 \mathrm{a}) .^{8}$

The theory is also capable of dealing with particularised scalar implicatures (Hirschberg 1991, among others), which are scalar implicatures generated by contextually salient alternatives. For instance, consider the following example.

$$
\text { Context: Mary got drunk. What did John do? }
$$

a. He smoked pot.

b. $\rightsquigarrow$ John didn't get drunk

The scalar implicature in (11b) is derived by negating the sentence He got drunk. This is a formal alternative to (11a), since the constituent got drunk is contextually salient in this example. ${ }^{9}$

\subsection{The problem of indirect scalar implicatures}

The structural theory of alternatives runs into a problem with so-called indirect implicatures, as pointed out by Romoli (2013). Indirect scalar implicatures are implicatures of sentences containing strong scalar items in downward entailing contexts like negation. The crucial property of these cases is that unlike the examples we have seen so far, the asserted sentence is structurally more complex than the problematic symmetric alternative, and hence the substitution procedure by Katzir (2007) automatically includes such symmetric alternatives as formal alternatives.

\footnotetext{
8 The definition of formal alternatives in (6) allows constituents that are salient in the context to be in the substitution source. One remaining question is whether one can make problematic symmetric alternatives salient in the context so that they can be formal alternatives. Katzir (2007) and Fox and Katzir (2011) discuss the following example.
}

(i) ??Yesterday John did some but not all of the homework and today he did some.

Sentence (i) is odd. Nonetheless, to the extent that it is acceptable, the intuition is that no inference is drawn from it. And indeed this is predicted by the closure condition above, which dictates that it is not possible to have just (2) in $C$ and not also have John did all of the homework. Consequently, the implicature that John did all of the homework is correctly not expected for (i).

9 An alternative neo-Gricean treatment of these particularised scalar implicatures is given in Hirschberg (1991), which builds on the lexical alternatives approach and develops the idea that sets of alternatives are created on an ad hoc basis according to what is relevant in context and are partially ordered by entailment. In this example, an ad hoc scale would give rise to an alternative that John smoked pot and got drunk. 
Consider (12a), for example. It has a scalar implicature in (12b), which is obtained by negating (13).
a. John didn't do all of the homework.
b. $\rightsquigarrow$ John did some of the homework

John didn't do any of the homework.

The structural approach wrongly predicts that this alternative cannot be negated, due to the presence of its symmetric alternative. Consider first the set of formal alternatives of (12a) that can be constructed according to the definition in (6). To avoid clutter in the examples, we will write alternatives in schematic form from now on, adopting the notation in Trinh and Haida (2015) (e.g., all = 'John did all of the homework').

$$
F(12 \mathrm{a})=\{\neg \text { all, } \neg \text { any, all, some }\}
$$

Here we would like to negate $\neg$ any to obtain the inference (12b), but we cannot, because we also have the symmetric alternative some, generated from (12a) by substituting the NegP in the assertion with its subconstituent VP, and substituting some for all within the latter, as shown in (15). ${ }^{10,11}$

$$
\begin{aligned}
& \text { [NegP not [vP John did all of the homework]] } \\
\longrightarrow & \text { [vP John did all of the homework] } \\
\longrightarrow & \text { [vP John did [some] of the homework] }
\end{aligned}
$$

subconstituent all/some

Given that some is in $C$, the negation of $\neg$ any contradicts the negation of some and hence cannot be negated. Note that it is not possible to use a subset $C^{\prime}$ of the formal alternatives containing $\neg$ any but not some, i.e. $C^{\prime}=\{\neg$ all, $\neg$ any, all $\}$, to derive the desired scalar implicature, because $C^{\prime}$ would violate the closure condition: some is both in $\mathrm{F}(12 \mathrm{a})$ and in the Boolean closure of $C^{\prime}$, given that $\llbracket$ some $\rrbracket=\llbracket \neg \neg$ any $\rrbracket$.

It should be noted here that Fox and Katzir (2011) assume an additional constraint on the set of formal alternatives that requires replacements to be performed only on focussed parts of the sentence. If focus is narrow enough, i.e. below negation, as in (16a) or (16b), then the offending alternative some cannot be generated, as negation cannot be replaced here.

a. John didn't [do all of the homework $]_{F}$.

b. John didn't do [all of the homework $]_{F}$.

Romoli (2013), however, points out that the scalar implicature is still observed, even when the focus is broad enough to include negation, as in the following example.

\footnotetext{
10 For expository purposes, we represent the subject in the VP-internal position and the verb in the inflected form, but nothing crucial hinges on this.

11 We use ' $\longrightarrow$ ' to indicate steps in a derivation of alternatives.
} 
What happened at school today?

[John (my favourite student) didn't do all of the homework $]_{F}$.

Thus the under-generation problem noted above persists for such cases. ${ }^{12}$

\subsection{The problem of particularised scalar implicatures}

A similar problem to that with (17) arises with the slightly more complex example involving a particularised implicature in (18), adapted from Trinh and Haida (2015).

Bill went for a run and didn't smoke. What did John do?

John went for a run.

$\rightsquigarrow$ John smoked

As evidence for this inference, notice that (18) sounds unnatural in a context in which it is known that John didn't smoke. This parallels the unnaturalness of sentences like (11) and (12a) in contexts in which their implicatures are false-ones in which John didn't do any homework and ones in which he did get drunk, respectively. ${ }^{13}$

The implicature of (18) could be derived in principle by negating $\neg$ smoke, or run $\wedge \neg$ smoke (which together with the assertion, run, entails smoke). But here again, the system proposed by Fox and Katzir (2011) undergenerates, for essentially the same reason as above. Assuming that the VPs in (18) are salient, $\neg$ smoke, run $\wedge \neg$ smoke, or both can be generated as formal alternatives, so we have:

$$
F(18) \supseteq\{\text { run, } \neg \text { run, smk, } \neg \mathbf{s m k}, \text { run } \wedge \text { smk, run } \wedge \neg \text { smk }\}
$$

\footnotetext{
12 This problem would not arise if only stronger members of $C$ could be negated, rather than non-weaker ones. However, there is independent evidence against this, as discussed in Sect. 1.2. Nonetheless, one might still wonder if one could exploit the fact that the two symmetric alternatives some and $\neg$ any differ in their relation to the assertion, insofar as the latter but not the former entails the assertion. That is, one could modify the conditions on contextual restriction in (8) in such a way that when there are symmetric alternatives, where one entails the assertion and the other does not, then context can disregard the latter. In other words, contextual restriction distinguishes between alternatives that are strictly stronger and alternatives that are logically independent. While this move could handle the problem with indirect scalar implicatures, it would run into trouble with cases like (18) involving particularised implicatures, discussed by Trinh and Haida (2015), where both symmetric alternatives entail the assertion. We turn to these in the next section.

13 Some speakers pointed out to us that for them (18) tends to have an ignorance inference that the speaker is unsure as to whether John smoked, rather than the scalar implicature that John smoked. We agree that this reading is possible and maybe even the most salient. However, in variants of (18), like (i), for which it is very plausible that the speaker is well informed about the relevant activity that she herself has engaged with, the scalar implicature tends to arise just as easily.
}

(i) Bill went for a run and didn't smoke.

I went for a run.

$\rightsquigarrow$ I smoked

Similarly, the use of explicit 'only' can make the reading with ignorance inferences disappear.

(ii) Bill went for a run and didn't smoke.

John only went for a run.

$\rightsquigarrow$ I smoked 
However, the closure condition on $C$ requires it to contain the symmetric alternative run $\wedge$ smoke, if $C$ contains run $\wedge \neg$ smoke, since $C$ must contain run itself and any formal alternative in its Boolean closure ( $\llbracket$ run $\wedge$ smoke $\rrbracket=$ 【run $\wedge \neg($ run $\wedge \neg$ smoke $) \rrbracket$.) For parallel reasons, if $C$ contains $\neg$ smoke, it must also contain the symmetric alternative smoke.

\section{The atomicity constraint and its problems}

\subsection{The atomicity constraint}

Adopting the structural approach to alternatives of Katzir (2007) and Fox and Katzir (2011), Trinh and Haida (2015) propose to impose one extra constraint on the construction of formal alternatives, the Atomicity Constraint. The Atomicity Constraint prohibits further substitutions on the expressions in the substitution source. Or to put it differently, the Atomicity Constraint effectively forces all constituents in the substitution source to be treated as if they were atomic lexical items, so substitutions within them are prohibited.

Let us see how this solves the problem of indirect scalar implicatures. Remember that the problem with (12a), repeated here as (20a), was that the procedure in Fox and Katzir (2011) allows us to construct both the needed alternative in (21a) and its symmetric alternative in (21b).

a. John didn't do all of the homework.

b. $\rightsquigarrow$ John did some of the homework

a. John didn't do some of the homework.

b. John did some of the homework.

The Atomicity Constraint correctly blocks the generation of the offending alternative (21b). To see this, consider how it could be derived. We know that the substitution source includes the atomic item some. Using this, let us try the following derivation.
a. [NegP not [vP John did all of the HW]]
b. vP John did all of the HW
c. VP John did [some] of the HW

subconstituent

*all/some

This derivation violates the Atomicity Constraint because after the second step, the subconstituent VP is now treated as an atom (as indicated by the box here), and its sub-part, all, cannot be replaced in the third step.

Note that first substituting some for all and substituting the newly formed [VP did some of the homework] for the NegP is not possible, simply because this VP is not in the substitution source. It is not a subconstituent of the assertion and it is not salient in the context. Consequently, the symmetric alternative (21b) (=some) is 
not generated, and the correct indirect scalar implicature can be generated by negating (21a) $(=\neg$ some $) .{ }^{14}$

Let us now also see how the particularised scalar implicature in example (18) is accounted for. The solution is essentially identical. Consider the first part of (18) in (23). Consider in particular the constituent $\alpha=$ [ $\mathrm{T}$, went for a run and didn't smoke]. Without the Atomicity Constraint, the offending alternative run $\wedge$ smoke could be derived as in (24):

$$
\begin{aligned}
\text { Bill } & {[\alpha \text { went for a run and didn't smoke }] } \\
& {[\text { John went for a run }]] } \\
\longrightarrow & {[\text { John } \mathrm{T} \text {, went for a run and didn't smoke }] } \\
\longrightarrow & {[\text { John } \mathrm{T} \text {, went for a run and [vP smoked }] }
\end{aligned}
$$

the prejacent

$\mathrm{T} / \alpha$

$* \mathrm{NegP} / \mathrm{smoked}$

The Atomicity Constraint rules out this derivation, as the T' in the second step is atomic and the replacement in the last step is forbidden. As a result, the formal alternatives do not include the offending alternative run $\wedge$ smoke and $C$ can be $\{$ run, run $\wedge \neg$ smoke $\}$, which gives rise to the correct scalar implicature.

We can also construct other formal alternatives from (23), in particular (25a) and (25b), based on the saliency of the VPs in (23).

$$
\begin{aligned}
& \text { a. John [ } \beta \text { didn't smoke] } \\
& \text { b. John }[\gamma \text { smoked }]
\end{aligned}
$$

Given the closure condition, we cannot have one of (25a) and (25b) in $C$ without having the other. However, this causes no problem, since neither is in the Boolean closure of $\{$ run, run $\wedge \neg$ smoke $\}$ and so they can be excluded.

\subsection{Problems}

We now show that there are still instances of the symmetry problem that the Atomicity Constraint does not account for. We discuss two sets of new data. One is based on variants of the example by Trinh and Haida (2015) in (18) with a particularised

\footnotetext{
14 What if this VP is contextually salient instead? Consider the following example.

(i) Two weeks ago, John did all of his homework. Last week, he did some. And this week, he didn't do all of his homework.

$\rightsquigarrow$ John did some of his homework
}

The discourse is perhaps not completely natural. However, if one can establish that the last sentence has the inference that John did some of the homework, this would not be predicted, since [VP John did some of the homework] is in the substitution source by virtue of having been uttered in the previous sentence, so the scalar implicature should be absent in this case.

Both Fox and Katzir (2011) and Trinh and Haida (2015) are aware of this potential problem, and they propose that contextually salient alternatives are only optionally in the substitution source (see Fox and Katzir 2011: fn. 23 and Trinh and Haida 2015: fn. 22). With this assumption, the scalar implicature is optionally derived for (i), which appears to be a correct prediction, as the scalar implicature could be seen as an optional inference. 
implicature. The other has to do with the indirect scalar implicatures that follow from gradable adjectives like full or empty under negation.

\subsubsection{Particularised scalar implicatures}

What is crucial for the explanation by Trinh and Haida (2015) of (18) is that the first part makes salient the conjunctive constituent $\alpha$ in the box in (26).

$$
\text { Bill }[\alpha \text { went for a run and didn't smoke] }
$$

This constituent allows us to construct the alternative in (27a), the negation of which, in conjunction with the prejacent, is the attested implicature that John did smoke. Crucially, given the Atomicity Constraint, the alternative John went for a run and smoked is not included, as shown in (27b).

$$
\begin{aligned}
& \text { a. } \longrightarrow \text { John }[\alpha \text { went for a run and didn't smoke }] \\
& \text { b. } \longrightarrow \text { John }[\alpha \text { went for a run and smoked }]
\end{aligned}
$$

The situation changes, though, when conjunction is absent or in a different place. Consider for instance (28), which has the same inference that John smoked. ${ }^{15}$

Bill went for a run. He didn't smoke. What did John do?

John went for a run.

$\rightsquigarrow$ John smoked

Example (28), however, does not contain the crucial conjunctive constituent. Consider the following constituents (28) makes salient:

$$
\text { Bill [ } \beta \text { went for a run }] \text {. He }[\gamma \text { didn’t }[\phi \text { smoke }]]
$$

Clearly none of $\beta, \gamma$, or $\phi$ will be of help: by substituting them for T' in (30) we can create the alternatives in (31); yet we cannot create a set of alternatives from (31) which will allow us to conclude that John smoked.

$$
\text { John }\left[\mathrm{T}^{\prime}\right. \text { went for a run ] }
$$

$$
\{\text { John }[\beta \text { went for a run }], \text { John }[\phi \text { smoked }] \text {, John }[\gamma \text { didn't smoke }]\}
$$

The desired scalar implicature would obtain if the alternative $\neg$ smoke is negated, but, as mentioned above, this cannot be done due to its symmetric counterpart smoke. And smoke cannot be excluded from $C$ due to the closure condition. In addition, crucially,

15 To make (28) more natural we could add another sentence as in (i).

(i) Bill went for a run. He lifted weights. He didn't smoke. What did John do? John went for a run. John didn't lift weights $\rightsquigarrow$ John smoked 
this time we do not have the conjunctive alternative run $\wedge \neg$ smoke, which allowed us to derive the implicature before. ${ }^{16}$

\title{
3.2.2 Gradable adjectives
}

Another instance of the symmetry problem that the Atomicity Constraint fails to account for involves indirect scalar implicatures generated by adjectives like full or empty under negation. Consider the following example, for instance. ${ }^{17}$

It's not the case that the glass is full.

a. $\rightsquigarrow$ The glass is not empty/is a bit filled

b. $\Varangle$ The glass is empty

This sentence has a robust inference that the glass is not empty and it does not implicate that the glass is empty. ${ }^{18}$ (33) presents the opposite pattern: it suggests that the glass is not completely filled and it doesn't suggest that it is full. ${ }^{19}$

It's not the case that the glass is empty.

a. $\rightsquigarrow$ The glass is not full/not completely filled

b. $\longleftrightarrow \rightarrow$ The glass is full

\begin{abstract}
$\overline{16}$ Fox and Katzir (2011) and Trinh and Haida (2015) allow contextually salient constituents to be optionally ignored. Would this help in any way here? It would appear to make things worse, because if the salient constituent didn't smoke is ignored, we will end up having the following set of alternatives: $C=\{$ run, smoke $\}$. From this set and the assertion we should conclude that John didn't smoke, which is the opposite of what we want to obtain.

17 The reason why we use the sentential negation form here rather than the more natural (i) is because, depending on one's assumptions about the LF of (i), the Atomicity Constraint can avoid the overgeneration problem we discuss below. Nonetheless, while (32) sounds less natural than (i), the judgments about (32a) and (32b) appear clear in both cases.
\end{abstract}

(i) The glass isn't full.

18 One might ask at this point whether this could be a presupposition rather than an implicature. While this is a possibility that we cannot exclude altogether, we think that the tests for presuppositionality, as imperfect as they are, suggest that this is not the case. For instance, a "Hey wait a minute!" response to (ia), as given in (ib), appears infelicitous (von Fintel 2004).

(i) a. The glass is full.

b. ??Hey wait a minute, I didn't know it wasn't empty!

Similarly, a sentence like (iia) doesn't appear to us to project the inference that the glass is not empty, or at least not more than a sentence like (iiia) projects the inference that some of the students came.

(ii) a. Is the glass full?

b. ? $\rightsquigarrow$ The glass is not empty

(iii) a. Did all of the students come?

b. ? $\rightsquigarrow$ Some of the students came

19 How can we be sure that an inference like (32b) is absent, given that it is actually compatible with the literal meaning of (32)? One argument comes from Hurford's Constraint (see Chierchia et al. 2012, among 
Trinh and Haida (2015), however, predict exactly the opposite for these cases. That is, they predict that a sentence like (32) or (33) should be able to have a scalar implicature like the one in $(32 b) /(33 b)$, and not the one in $(32 a) /(33 a)$. Similar cases can be created with other adjectives: both (34) and (35) suggest the inferences in (a) but not those in (b).

(34) It's not the case that a tie is required.

a. $\rightsquigarrow$ A tie is allowed

b. $\leftrightarrow \rightarrow$ A tie is mandatory

(35) It's not the case that Mary's promotion is certain.

a. $\rightsquigarrow$ Mary's promotion is possible

b. $\longleftrightarrow$ Mary's promotion is impossible

Notice that if the sentence It is not the case that the glass is empty were an alternative for (32), or It is not the case that the glass is full were an alternative for (33) (and similarly for the other cases), the desired inferences would be generated. But, as we show below, the Atomicity Constraint prohibits these sentences from being a formal alternative. So the first question for Trinh and Haida is how to derive the actually observed inferences.

There is, in fact, a way to derive the observed scalar implicature, once we look more closely at the syntax/semantics of the sentences above. It is often assumed that a sentence with a positive adjective involves the silent positive morpheme POS (Bartsch and Vennemann 1975; Stechow 1984; Kennedy 1999), as illustrated in (36) for (32).

[it is not the case that [the glass is [ POS full ]]]

The main function of POS is to introduce the standard of fullness in the context, which is typically the maximal fullness for the glass (Kennedy 2007; McNally 2011). It is also assumed that it occupies the same syntactic position as other modifiers of gradable adjectives, like partly, half, etc. Then it is not too far-fetched to assume under the structural theory of alternatives that POS can be replaced with these modifiers to give rise to alternatives of the following form:

[it is not the case that [the glass is [ half/partly full ]]]

These alternatives are stronger, and negating them would gives us something that resembles the inference that the glass is not empty.

Footnote 19 continued

others). One test would be to construct a sentence where the second disjunct entails the first unless the first disjunct can have the inference that we are investigating. Given these assumptions, the following contrast indicates that the inference is not there.

(i) a. ??Either it's not true that the glass is full or it is partially filled.

b. Either the glass is empty or it is partially filled. 
So far so good. However, if this were the source of the scalar inference, the following sentences should also have similar scalar implicatures, which does not seem to be the case.

(38) a. This neighbourhood is not safe.

y $\rightarrow$ This neighbourhood is not dangerous

b. John is not tall.

$\varkappa \rightarrow$ John is not small

c. The glass is not transparent.

« The glass is not opaque

One might wonder if the different scale structures of these adjectives and their (in)compatibility with modifiers like partly and half might explain the difference here. However, notice that we picked three adjectives cutting across the absolute/relative distinction and differing with respect to their scale structure: safe has an upper closed scale, transparent has a fully closed one, and tall a fully open scale (Kennedy 2007, among others). Thus the scale structure is not a relevant factor. In particular, transparent could combine with the same kind of modifiers as full but it still does not give rise to the scalar implicature indicated above.

In addition to this problem of explaining the variation among gradable adjectives, the system in Trinh and Haida (2015) also predicts unwanted inferences. More specifically, it predicts the inference in (32b) for (32), due to the alternative $\neg$ empty which is obtained by simple lexical substitution of empty for full. This problem persists regardless of whether POS is assumed or not.

$$
\begin{aligned}
& \text { It's not the case that [the glass is [ POS full ]] } \\
& \longrightarrow \text { It's not the case that [the glass is [ POS empty ]] }
\end{aligned}
$$

full/empty

This inference would be correctly blocked if the alternative empty was available, but as remarked above, the Atomicity Constraint would prohibit empty from becoming a formal alternative. That is, the following derivation is illicit.

$$
\begin{aligned}
& \text { It's not the case that [the glass is [ POS full ]] } \\
& \longrightarrow \text { [the glass is [ POS full ]] } \\
& \longrightarrow \text { [the glass is [POS empty ]] }
\end{aligned}
$$

The same can be shown for the cases in (34) and (35). Notice that this is precisely what gave Trinh and Haida (2015) the solution for the earlier examples (12a) and (18). Not having the Atomicity Constraint, Fox and Katzir (2011) do not run into this problem, but, as explained above, they fail to account for (12a) and (18). These observations are, therefore, connected in that the solution by Trinh and Haida (2015) for one set of cases creates a problem for the other. ${ }^{20}$

20 One might wonder if an alternative like The glass is not partly full could be used to block the unwanted inference for Trinh and Haida (2015). Indeed, if such an alternative is present in the sets of alternatives, it makes the alternative The glass is not empty not excludable. However, since it is not in the Boolean closure, 


\subsection{Section summary}

To summarise, the Atomicity Constraint by Trinh and Haida (2015) faces two issues. One problem comes from variants of the type of case they proposed as problematic for Fox and Katzir (2011). Without the conjunction in the 'right' place, the crucial alternative run $\wedge \neg$ smoke cannot be in the substitution source. The other problem is the indirect scalar implicatures of gradable adjectives like full or empty. Here, the Atomicity Constraint backfires as it prevents the symmetric alternative empty to be in the substitution source, resulting in the wrong scalar implicature. The Atomicity Constraint, therefore, only solves the original examples (12a) and (18), but it is not a general solution.

\section{Two more problems for the structural approach}

In the this section, we will discuss two further problems that illustrate the general difficulty of deriving the correct set of relevant alternatives under the structural approach, independently of the Atomicity Constraint.

\subsection{Too few lexical alternatives}

One potential problem is that of undergeneration, where the needed formal alternative cannot be generated under the assumptions of the structural approach to alternatives. For instance, in Japanese, deontic possibility and necessity are expressed by constructions that are structurally clearly different. Consider the following examples.

$$
\text { John-wa ki-te yoi. }
$$

John-TOP come-GERUND good

'John is allowed to come.'
a. John-wa ko-naku-te-wa nar-anai/ike-nai.
John-TOP come-NEG-GERUND-TOP become-NEG/go-NEG
'John must come.'
b. John-wa kuru hitsuyoo-ga aru.
John-TOP come necessity-NOM exist
'John needs to come.'

Sentence (41) expresses deontic possibility with the predicate yoi, which is morphologically an adjective. By contrast, the sentences expressing deontic necessity in (42) do not involve adjectives. Specifically, (42a) involves a verbal stem (either nar- or ike-) with the negative suffix -(a)nai. This makes the main predicate morphologically more complex than in (41). Generally, the structural approach to alternatives assumes that adding negation complicates the structure, so it is unlikely that (42a) can be derived

Footnote 20 continued

there is no reason to assume that it must be in $C$. $C$ is allowed to simply be \{The glass is not full, The glass is not empty\}, and the unwanted inference is still predicted in this case. 
from (41) by substitution and deletion alone. ${ }^{21}$ In addition, the topic marking on the gerundive subject here is obligatory, while adding it would make (41) unacceptable. This could be taken as suggesting that the gerundive clause is in syntactically distinct positions in (41) and (42a). Similarly, (42b) is unlikely to be derivable from (41) with substitution and deletion. It involves an existential construction with the existential verb $a r$-, where the subject is not a gerundive clause but a nominal hitsuyoo 'necessity' with a complement clause.

Despite this structural difference, however, (41) has the same scalar implicature as the English translation, i.e. that John is not required to come. As evidence for this inference, we observe that (41), like its English counterpart, is very unnatural in a context in which John is required to come. However, it is not at all clear how (42) could be generated in the structural approach from (41) (which presumably has a structure like (43)).

$$
\text { [John-wa] [ki-te [yoi]]. }
$$

A possible response to this might be to assume that there actually is a grammatical alternative to (41) that expresses deontic necessity (e.g. *[John-wa] [ki-te [doi]]) but is made unacceptable and practically unusable for some independent reasons, to which computation of scalar implicatures is somehow oblivious. ${ }^{22}$ Then, the desired scalar implicature could be generated based on this unacceptable sentence. However, a solution like this commits one to nontrivial assumptions about the theory of the lexicon and the acquisition of lexical items (cf. Schlenker 2008).

\subsection{Too many lexical alternatives}

For the symmetry problem created by some but not all, the solution under the structural approach to alternatives crucially relies on what is and is not lexicalised. In particular, it is important that there be no constituent in the lexicon of the same or less structural complexity as some and all and with the meaning 'some but not all'.

\footnotetext{
21 One might wonder if the suffixal nature of the negation -(a)nai in (42a) allows (42a) to be a structural alternative to (41). That is, if substitution is an operation over (phonological) words rather than over morphemes, nar-anai/ike-nai would be just as complex as yoi. However, observe that (i) has a scalar implicature that John met some of the students, just like its English translation ((i) has a (contrastive) topic marking on the quantified object to facilitate the wide scope reading of the negation).
}

(i) John-wa subete-no gakusei-to-wa aw-anakatta.

John-TOP all-GEN student-with-TOP meet-NEG.PAST

'John didn't meet all of the students.'

If the negated verb aw-nakatta 'didn't meet' were as structurally complex as the positive counterpart atta 'met', then (ii) would be one of the structural alternatives for (i), and would wrongly block the observed scalar inference.

(ii) John-wa nanninka-no gakusei-to-wa at-ta.

John-TOP some-GEN student-with-TOP meet-PAST

'John met some of the students.'

22 Thanks to Andreas Haida (p.c.) for suggesting a possibility along these lines, and related discussion. 
Swanson (2010), however, points out that there do appear to be other scalar items whose symmetric alternative is lexicalised. He raises examples like the following, where the scalar items are permitted and sometimes.
a. Going to confession is permitted.
b. $\rightsquigarrow$ Going to confession is optional
c. $\nLeftarrow$ Going to confession is required
a. The heater sometimes squeaks.
b. $\rightsquigarrow$ The heater intermittently/occasionally squeaks
c. $\leftrightarrow \rightarrow$ The heater constantly squeaks

The sentences in (b) and (c) appear to be symmetric alternatives, analogous to just some and all, yet (a) yields the implicature indicated in (b) — and never that in (c) exactly analogous to the case with some. This instantiation of the symmetry problem appears to straightforwardly resist the structural approach, both in its original version by Katzir (2007), Fox and Katzir (2011) and in the modified version by Trinh and Haida (2015). ${ }^{23}$ As Swanson (2010) himself suggests, one could try to supplement the

\footnotetext{
23 An anonymous reviewer of this journal has pointed out to us that there is some evidence against the assumption above that alternatives like intermittently, occasionally, and optional are in fact symmetric to their counterparts required and always. The examples below suggest, instead, that the meaning component that turns the former into symmetric alternatives of the latter is in turn cancellable. That is, they appear themselves derived via scalar strengthening.
}

(i) a. An end-of-night greasy burger is optional, if not obligatory.

b. Affixation of the possessed noun is optional, if not obligatory.

c. It would also be well to make [it] optional, if not obligatory.

(ii) a. Her mind was occasionally, if not always, crazed.

b. Most vascular surgeons occasionally, if not always, patch selected carotid arteries.

(iii) a. HiSeq will probably have this same problem, intermittently, if not persistently.

On the other hand, as pointed out to us by Matt Mandelkern (p.c.), there is also evidence against this hypothesis, coming from a contrast like the one in (iv) vs. (v) below, where the former sounds acceptable but the latter is clearly degraded. A similar contrast can be created for intermittently. These contrasts appear therefore to point to evidence in favour of the assumption that alternatives like optional and intermittently are indeed symmetric alternatives of required and always.

(iv) It's permitted and moreover obligatory.

(v) \#It's optional and moreover obligatory.

Finally, notice that some of the examples above are more convincing than others. For instance, it is unclear that occasionally really has the problematic meaning sometimes but now always; it is unclear whether (vi) is contradictory, as would be predicted by such meaning. Intermittently, on the other hand, appears more convincingly upper-bounded: (vii) does appear to be contradictory.

(vi) All logicians occasionally make errors, and some do so constantly.

(vii) ??It rains intermittently everywhere in the Netherlands, and in Amsterdam it rains constantly.

We leave further investigation of these examples and the role they play for the structural approach to future research. 
theory with a constraint that excludes the problematic lexical items in some principled way, e.g., by resorting to their relative low frequency. However, it appears not obvious to us how to integrate these other factors into the structural approach.

\section{Alternative approaches}

In addition to the structural theory of alternatives, there are two other theories on the market that we know of that address the symmetry problem: the Monotonicity Constraint (Horn 1989; Matsumoto 1995) and the interaction of informativity and complexity in the Rational Speech Acts (RSA) framework proposed by Bergen et al. (2016). The former has been criticised extensively in Katzir (2007), so we refer the reader to that for discussion. In this section, we will briefly review the RSA approach and what we see as its limitations.

\subsection{The RSA approach}

In a recent paper, Bergen et al. (2016) propose an alternative approach to the symmetry problem, which, they claim, provides a 'straightforward solution' to the problem. ${ }^{24}$ In this subsection, we first summarize the gist of their account in relation to the symmetry problem and the cases which it can successfully cover. We will then move to cases which, as far as we can see, remain problematic for this account. We conclude that the RSA approach, as it stands, does not provide a full solution to the symmetry problem either.

\subsubsection{The account: complexity and relative informativeness}

Bergen et al. (2016) adopt and extends the RSA framework (Frank and Goodman 2012; Goodman and Stuhlmüller 2013). In our discussion, we will not review the details of this approach (which can be found in the texts cited) but focus instead on the crucial ingredients that play a role in a solution to the symmetry problem. ${ }^{25}$

The following three assumptions are particularly relevant: (i) while there are sets of alternatives for scalar implicature computation, there is no notion of 'scalar' alternative; (ii) complexity has a cost; and (iii) a notion of 'relative' informativity is a factor in deciding among alternatives. Recall that the structural approach uses no real notion of 'scale' and the cost of complexity arises as a by-product of the way the alternative algorithm is defined. Therefore, among (i)-(iii), the structural and RSA approaches differ only with respect to (iii).

\footnotetext{
24 Thanks to Noah Goodman and Leon Bergen for discussion on the points in this subsection.

25 See Bergen et al. (2016) and Goodman and Stuhlmüller (2013) for a more detailed introduction to the RSA approach. For application of this approach to other phenomena see Kao et al. (2014a), Kao et al. (2014b) and Lassiter and Goodman (2013).
} 
To illustrate the way that the account by Bergen et al. (2016) works, consider (46) and its set of alternatives (47), which, unlike in the structural approach, include both all and just some (or some-but-not-all) alternatives.

John saw some of the students.

\{John saw all of the students, John saw just some of the students

Let us partition the space of logical possibilities as worlds in which John met none of the students, worlds where John met all of the students, and ones where John met some but not all of the students, indicated respectively as $\neg \exists, \forall$, and $\exists \wedge \neg \forall$. Thus, the informativeness of each of the two alternatives in (47) is the same and greater than that of the assertion, some. ${ }^{26}$ What makes the difference here is that RSA reasoning turns on the utility of utterances for the speaker, and this in turn is affected by both informativeness and cost. In this case, the just some alternative is penalised by complexity when it comes to determining its utility, thus creating a situation where the asserted alternative some has a higher conditional probability in a $\exists \wedge \neg \forall$ world than in a $\forall$ world, due to the lower complexity and thus higher utility of all, relative to some, in a $\forall$ world. This in turn gives the hearer reason to conclude that the speaker's observed world is more likely to be a $\exists \wedge \neg \forall$ world. Further higher-order iterations of this reasoning strengthen this conclusion.

As we know from above, in the case of indirect scalar implicatures arising from sentences like (48) there is, however, no difference in complexity between the two corresponding symmetric alternatives in (49). ${ }^{27}$ And therefore in this case complexity cannot be used to break symmetry (and this was in fact one of the problematic cases for the structural account by Fox and Katzir (2011), which required the addition of Atomicity by Trinh and Haida (2015)).

John didn't see all of the students.

\{John saw some of the students, John saw none of the students\}

While it cannot rely on complexity for the case of (48), the RSA approach can, however, exploit the other ingredient mentioned above, namely relative informativeness, to create an asymmetry between the two alternatives. To illustrate, consider again a partition of the space of possibilities into $\{\neg \exists, \forall, \exists \wedge \neg \forall\}$. Here we have a situation where the unwanted some alternative is not more complex than the none alternative, which we would want to negate to obtain the intuitively correct inference. Therefore complexity cannot be what breaks symmetry. Instead, it is the relative uninformativeness of some vis-a-vis not all when compared to that of none vis-a-vis not all that

\footnotetext{
26 This is because while the latter is true in both $\exists \wedge \neg \forall$ and $\forall$ worlds, all is only true in $\forall$ worlds and just some only in $\exists \wedge \neg \forall$ ones.

27 One could also consider the problematic alternative John saw some students to be less complex than the other, John saw none of the students. This depends on whether we consider the latter alternative to be decomposed as John didn't see some of the students and whether we consider the notion of complexity to reflect syntactic structure (as opposed to just utterance length as measured in number of words). While there are compelling arguments for the decomposition of none as in not some (see Sauerland 2000, among many others), this is certainly not an assumption that Bergen et al. (2016) have to make, and they do not appear to be making it, so we will put it aside here.
} 
makes the difference. This creates a situation where the conditional probability of not all for the speaker in $\exists \wedge \neg \forall$ worlds becomes greater than in $\neg \exists$ ones. In virtue of this, the hearer can conclude that it is more likely that the speaker has observed a $\exists \wedge \neg \forall$ situation than a situation $\neg \exists$, when they heard not all. Again, further iterations of this reasoning strengthens this conclusion.

Finally, the approach also makes good predictions in the other problematic case for the structural approach, namely sentences like (50), which, as discussed, intuitively gives rise to the inference that the glass is not empty.

(50) The glass is not full.

(51) $\{$ The glass is not empty, The glass is empty $\}$

To illustrate, consider the alternatives of (50) in (51), and assume a partition of the logical space in which either the glass is empty, it is neither full nor empty, or it is full: $\{$ EMPTY, $\neg$ EMPTY $\wedge \neg$ FULL, FULL\}. Given these assumptions, the two alternatives differ in informativeness, with not empty being true in both FULL and $\neg$ EMPTY $\wedge \neg$ FULL worlds and with empty only being true in EMPTY worlds. And, in addition, not empty is clearly more complex than empty. This creates a situation in which not empty is less likely to be used to communicate $\neg$ FULL $\wedge \neg$ EMPTY than empty is used to communicate EMPTY. And again, this then is the reason why the listener will correctly conclude that the speaker wanted to communicate $\neg$ FULL $\wedge \neg$ EMPTY rather than EMPTY when using not full.

In sum, the RSA approach, by combining a notion of complexity with one of relative informativity, can account for the cases of direct and indirect scalar implicatures, as well as the case of gradable adjectives under negation in (50). There are, however, a number of problems we can see for this approach, to which we turn in the next subsection.

\subsubsection{Problematic cases}

Starting from a general consideration, Bergen et al. (2016), unlike Fox and Katzir (2011), do not provide a general theory of alternatives and instead leave it vague how, in any particular instance, sets of alternatives are chosen. As they say, they assume that the relevant sets of alternatives are selected on a 'case by case' basis. In general, their approach "allows arbitrary sets of grammatical utterances to be considered as alternatives" (Bergen et al. 2016: p. 14). If this is the case, however, they need to provide a way in which the arbitrary sets of chosen alternatives avoid including the 'wrong' symmetric alternative to the exclusion of the other, giving rise to unattested inferences. $^{28}$

That is to say, if the model really allows for arbitrary sets of alternatives, what is it that prevents us from choosing the alternatives in (52) for (46), which presumably

\footnotetext{
28 Recall that this problem was avoided for the structural approach by adopting a notion of relevance and the closure condition in (8).
} 
would incorrectly predict the inference from (46) that John saw all of the students? And similarly, what prevents (48) from only having the alternatives in (53), incorrectly predicting the inference that John saw none of the students ${ }^{29}$

\{John saw just some of the students

\{John saw some of the students

On the other hand, the RSA approach can derive the attested scalar implicatures, while allowing symmetric alternatives among the possible alternative utterances the speaker might have used. This provides suggestive evidence that "every grammatical sentence in a language can be considered as an alternative during pragmatic reasoning" (Bergen et al. 2016: p. 10). It seems unrealistic to us to suppose that conversational participants always reason over all possible alternative utterances, relative to a space of possible observed states of affairs. But if this can indeed be maintained, then the problem above would not arise.

Aside from this general consideration regarding the choice of the alternative sets, we think there are more specific issues with the RSA account of the symmetry problem. The general shape of the problematic cases that we discuss below is the following. If we take a step back to see how the RSA model can deal with the three cases discussed, we see that unwanted alternatives do not affect the reasoning process to the extent that they have a lower utility vis-a-vis the assertion, when compared to the utility of the attested alternative vis-a-vis the assertion. More concretely, the approach was successful with the cases above because the symmetry between alternatives was broken either by complexity, or relative informativity, or a combination of both. Thus, if we find cases where the symmetric alternatives have comparable relative utility with respect to both complexity and relative informativity, we should expect symmetry again.

To make this idea more concrete, suppose for a moment that just some in (46) was no more costly than the alternatives some and all. In that case, the presence of just some as an alternative would affect the conditional probability that the speaker would use just some in a $\exists \wedge \neg \forall$ situation to the same extent that the presence of all would in a $\forall$ situation. The hearer would be left unable to infer which state holds-whether $\exists \wedge \neg \forall$ or $\forall$. Of course the problem in this case does not arise, precisely because just some is more complex than all. However, we shall now discuss three cases where the set of alternatives does create this kind of symmetry situation.

First, consider an utterance of (48) in a context in which it is relevant whether John saw many of the students. In such a case, we intuitively conclude from (48) that John saw many of the students. Let us consider a model then where the alternatives are as in (54) and consider the space of possibilities, $\{\neg \exists, \exists \wedge \neg$ MANY, MANY $\wedge \neg \forall, \forall\}$ :

\{John saw some of the students, John saw many of the students, John didn't see many of the students, John saw none of the students, John saw all of the students

\footnotetext{
29 They could assume that the reasoning applies only to alternatives that are made salient in the context. But then, it is unclear what makes all apparently automatically salient when some is uttered, even where just some has been made salient by the previous utterance, as discussed above in footnote 7 and extensively in Fox and Katzir (2011).
} 
Here is a model where the unwanted alternative, many, has equal (if not higher) relative utility vis-a-vis the assertion in MANY $\wedge \neg \forall$ situations, when compared to not many vis-a-vis the assertion in $\exists \wedge \neg$ MANY situations. This means that the hearer is unable to decide whether it is more likely that the speaker intended to convey a MANY $\neg \neg \forall$ interpretation than a $\exists \wedge \neg$ MANY one. Thus the hearer would not be able to conclude that the intended interpretation was that John saw many but not all of the students.

In the same way, Bergen et al. (2016) make incorrect predictions for the original case by Trinh and Haida (2015), repeated in (55), where the alternatives are also symmetric in terms of informativity (e.g. Bill ran and didn't smoke and Bill ran and smoked). If anything, given that the latter is less complex, they predict the opposite inference of what is in fact attested.

Bill ran and didn't smoke.

John ran.

$\rightsquigarrow$ John smoked

In addition, while we saw that the RSA approach makes correct predictions for the adjective case in (50), it doesn't account for the fact observed above that other adjectives do not give rise to the corresponding inferences, as illustrated again in (56). That is, for the same reason that this approach correctly predicts the inference of (50) that the glass is not empty, it incorrectly predicts the inferences below.
a. This neighbourhood is not safe.
$\leftrightarrow \rightarrow$ This neighbourhood is not dangerous
b. John is not tall.
$\not \rightarrow$ John is not small
c. The glass is not transparent. $\leftrightarrow \rightarrow$ The glass is not opaque

Finally, notice that the RSA approach in principle also has a problem with the cases by Swanson that we discussed in Sect. 4.2 above. That is, for a sentence like (57) we would have to consider the alternatives in (58). Yet for the RSA approach as for the structural approach, intermittently is not more complex than always in any obvious way, and we therefore cannot appeal to complexity to break the symmetry here. The RSA approach can however appeal to a notion of frequency as part of what counts as 'costly' in an utterance; it would make the intermittently alternative more costly and therefore break the symmetry in the desired way. ${ }^{30}$ In other words, the RSA approach, like the structural one, needs to appeal to factors other than linguistic complexity to account for cases like (57), but there is arguably more scope within a framework that relies on cost rather than mere complexity to do so.

The heater often squeaks.

\{The heater always squeaks, the heater intermittently squeaks\}

\footnotetext{
30 And in fact there is some evidence in the literature that less frequent words are harder to retrieve (Forster and Chambers 1973; Levelt 1989, among others), which could support this idea of treating them as costly.
} 
In sum, the RSA approach provides an interesting perspective on the symmetry problem, in particular by combining a notion of complexity with a notion of relative informativity in order to constrain the alternatives for implicature computation. As we sketched out above, however, as it stands, it does not provide a full solution to the symmetry problem

Before closing this section, let us briefly consider the option of integrating the notion of relative informativity from the RSA approach into the structural approach of Katzir (2007) and Fox and Katzir (2011). That is to say, let us consider adding to the latter the condition that symmetry can also be broken by informativity in the sense above. Putting aside the issue as to whether this addition would be more stipulative than explanatory, the resulting account would do better than either the structural approach or the RSA approach alone. It would have no problem with the simple indirect scalar implicature case in (48) and no problem with the gradable adjective case in (50). Still, this version of the structural approach would have problems with Trinh and Haida's original and modified cases, as well as the case of intermediate indirect scalar implicatures in (48) with alternatives like (54). Adding this notion of informativity, therefore, is still not enough to solve all the various problematic cases discussed above. ${ }^{31}$

\section{Concluding remarks}

The structural approach to alternatives advocated by Katzir (2007) and Fox and Katzir (2011) is arguably the most developed and systematic account of the symmetry problem that is available in the current literature. We argued that while it successfully accounts for certain instantiations of the symmetry problem, it does not handle others. In particular, we discussed three problems: (i) the problem of indirect and particularised scalar implicatures, (ii) the problem of too few lexical alternatives, and (iii) the problem of too many lexical alternatives. We argued that the Atomicity Constraint by Trinh and Haida (2015) does not constitute a general solution to the first problem. Moreover, such a constraint has little to say about the other issues. Therefore, we concluded that the structural approach, in its current form, does not fully solve the symmetry problem. We also considered briefly the RSA approach by Bergen et al. (2016). As we discussed, however, this approach also appears unable to solve certain problematic cases we discussed for the structural approach.

In sum, while we think that a lot of progress has been made on theories of alternatives for scalar implicatures since Gazdar's (1979) statement that "scales are in some sense given to us" (Gazdar 1979: p. 58), problematic cases above indicates that the symmetry problem still remains as an important open challenge for current theories.

Acknowledgements For invaluable discussion and feedback we thank Leon Bergen, Danny Fox, Noah Goodman, Andreas Haida, Roni Katzir, Matt Mandelkern, Tue Trinh, and Wataru Uegaki. Work on this project was partially supported by the British Academy Small Grant SG-153-238 to Jacopo Romoli and Yasutada Sudo and by the Leverhulme Trust grant RPG-2016-100 to Jacopo Romoli.

Open Access This article is distributed under the terms of the Creative Commons Attribution 4.0 International License (http://creativecommons.org/licenses/by/4.0/), which permits unrestricted use, distribution,

31 See also fn. 10 for a different way of modifying the structural approach by adding a notion of informativity. 
and reproduction in any medium, provided you give appropriate credit to the original author(s) and the source, provide a link to the Creative Commons license, and indicate if changes were made.

\section{References}

Bartsch, R., and T. Vennemann. 1975. The grammar of adjectives and comparison. Linguistische Berichte 20: 19-32.

Bergen, L., R. Levy, and N. Goodman. 2016. Pragmatic reasoning through semantic inference. Semantics and Pragmatics 9(20). http://semprag.org/article/view/sp.9.20/pdf.

Chemla, E., and B. Spector. 2011. Experimental evidence for embedded scalar implicatures. Journal of Semantics 28(3): 359-400.

Chierchia, G., D. Fox, and B. Spector. 2012. Scalar implicature as a grammatical phenomenon. In Semantics: An international handbook of natural language meaning, ed. C. Maienborn, K. von Heusinger, and P. Portner, 2297-2331. Berlin: de Gruyter.

Crnic, Luka, L., E. Chemla, and D. Fox. 2015. Scalar implicatures of embedded disjunction. Natural Language Semantics 23(4): 271-305.

Fine, K. 2017. Truth-maker semantics. In A companion to the philosophy of language, 2nd ed, ed. B. Hale. London: Wiley Blackwell.

Forster, K., and S. Chambers. 1973. Lexical access and naming time. Journal of Verbal Learning and Verbal Behavior 12: 627-635.

Fox, D. 2007. Free choice and the theory of scalar implicatures. In Presupposition and implicature in compositional semantics, ed. U. Sauerland and P. Stateva, 71-120. London: Palgrave Macmillan.

Fox, D., and R. Katzir. 2011. On the characterization of alternatives. Natural Language Semantics 19(1): 87-107.

Frank, M., and N. Goodman. 2012. Predicting pragmatic reasoning in language games. Science 336(6084): 998.

Gazdar, G. 1979. Pragmatics: Implicature, presupposition and logical form. New York: Academic Press.

Goodman, N., and A. Stuhlmüller. 2013. Knowledge and implicature: Modeling language understanding as social cognition. Topics in cognitive science 5: 173-184.

Hirschberg, J.B. 1991. A theory of scalar implicature. New York: Garland.

Horn, L. 1972. On the semantic properties of logical operators in English. Ph.D. thesis, UCLA.

Horn, L. 1989. A natural history of negation. Chicago: The University of Chicago Press.

Kao, J., L. Bergen, and N. Goodman. 2014a. Formalizing the pragmatics of metaphor understanding. Proceedings of the annual meeting of the Cognitive Science Society 36. London: Cognitive Science Society.

Kao, J., J. Wu, L. Bergen, and N. Goodman. 2014b. Nonliteral understanding of number words. Proceedings of the National Academy of Sciences 111 (33): 12002-12007.

Katzir, R. 2007. Structurally-defined alternatives. Linguistics and Philosophy 30 (6): 669-690.

Katzir, R. 2014. On the roles of markedness and contradiction in the use of alternatives. In Semantics, pragmatics and the case of scalar implicatures, ed. S. Pistoia-Reda, 40-71. London: Palgrave Macmillan.

Kennedy, C. 1999. Projecting the adjective: The syntax and semantics of gradability and comparison. New York: Garland Press.

Kennedy, C. 2007. Vagueness and grammar: the semantics of relative and absolute gradable adjectives. Linguistics and Philosophy 30: 1-45.

Kroch, A. 1972. Lexical and inferred meanings for some time adverbs, Quarterly Progress Report of the Research Laboratory of Electronics 104. Cambridge, MA: MIT.

Lassiter, D., and N. Goodman. 2013. Context, scale structure, and statistics in the interpretation of positiveform adjectives. In Proceedings of SALT 23, ed. T. Snyder, 587-610. LSA. Washington, D.C.

Levelt, W. 1989. Speaking: From intention to articulation. Cambridge, MA: MIT Press.

Matsumoto, Y. 1995. The conversational condition on Horn scales. Linguistics and Philosophy 18 (1): 21-60.

McNally, L. 2011. The relative role of property type and scale structure in explaining the behavior of gradable adjectives. In Vagueness in Communication, ed. R. Nouwen, R. van Rooij, U. Sauerland, and H.-C. Schmitz, 151-168. Berlin: Springer.

Romoli, J. 2013. A problem for the structural characterization of alternatives. Snippets 27: 14-15. 
Sauerland, U. 2000. No 'no': on the crosslinguistic absence of a determiner 'no'. In Proceedings of the Tsukuba Workshop on Determiners and Quantification, ed. H. Suzuki, 415-444. Tsukuba, Japan: Tsukuba University.

Sauerland, U. 2004. Scalar implicatures in complex sentences. Linguistics and Philosophy 27(3): 367-391. Schlenker, P. 2008. Presupposition projection: Explanatory strategies. Theoretical Linguistics 34(3): 287316. https://doi.org/10.1515/THLI.2008.021.

Soames, S. 1982. How presuppositions are inherited: a solution to the projection problem. Linguistic Inquiry 13(3): 483-545.

Spector, B. 2007. Aspects of the pragmatics of plural morphology: On higher-order implicatures. In Presupposition and implicature in compositional semantics, ed. U. Sauerland and P. Stateva, 243-281. Basingstoke: Palgrave.

Spector, B. 2016. Comparing exhaustivity operators. Semantics \& Pragmatics 9(11): 1-33.

Swanson, E. 2010. Structurally defined alternatives and lexicalizations of XOR. Linguistics and Philosophy 33(1): 31-36.

Trinh, T., and A. Haida. 2015. Constraining the derivation of alternatives. Natural Language Semantics 25(4): 249-270.

van Rooij, R., and K. Schulz. 2004. Exhaustive interpretation of complex sentences. Journal of Logic, Language and Information 13(4): 491-519.

van Rooij, R., and K. Schulz. 2006. Pragmatic meaning and non-monotonic reasoning: The case of exhaustive interpretation. Linguistics and Philosophy 29(2): 205-250.

von Fintel, K. 2004. 'Would you believe it? The King of France is back! Presuppositions and truth-value intuitions. In Descriptions and Beyond, ed. M. Reimer and A. Bezuidenhout, 315-341. Oxford: Oxford University Press.

von Stechow, A. 1984. Comparing semantic theories of comparison. Journal of Semantics 3(1-2): 1-77. 